$10-4-2004$

\title{
Angular Dependence of the Dipole-Dipole Interaction in a Nearly One-Dimensional Sample of Rydberg Atoms
}

Thomas J. Carroll

Ursinus College, tcarroll@ursinus.edu

Katharine Claringbould

Anne Goodsell

M.J. $\operatorname{Lim}$

Michael W. Noel

Follow this and additional works at: https://digitalcommons.ursinus.edu/physics_astro_fac

Part of the Atomic, Molecular and Optical Physics Commons

Click here to let us know how access to this document benefits you.

\section{Recommended Citation}

Carroll, Thomas J.; Claringbould, Katharine; Goodsell, Anne; Lim, M. J.; and Noel, Michael W., "Angular Dependence of the DipoleDipole Interaction in a Nearly One-Dimensional Sample of Rydberg Atoms" (2004). Physics and Astronomy Faculty Publications. 1.

https://digitalcommons.ursinus.edu/physics_astro_fac/1

This Article is brought to you for free and open access by the Physics and Astronomy Department at Digital Commons @ Ursinus College. It has been accepted for inclusion in Physics and Astronomy Faculty Publications by an authorized administrator of Digital Commons@ Ursinus College. For more information, please contact aprock@ursinus.edu. 


\title{
Angular Dependence of the Dipole-Dipole Interaction in a Nearly One-Dimensional Sample of Rydberg Atoms
}

\author{
Thomas J. Carroll, Katharine Claringbould, Anne Goodsell, ${ }^{*}$ M. J. Lim, ${ }^{\dagger}$ and Michael W. Noel \\ Department of Physics, Bryn Mawr College, Bryn Mawr, Pennsylvania 19010, USA
}

(Received 22 May 2004; published 4 October 2004)

\begin{abstract}
Atoms in an ultracold highly excited sample are strongly coupled through the dipole-dipole interaction. In an effort to understand and manipulate the complicated interactions in this system we are investigating their dependence on the relative orientation of the dipoles. By focusing a $480 \mathrm{~nm}$ beam from a tunable dye laser into a magneto-optical trap, we produce a nearly one-dimensional sample of Rydberg atoms. The trap lies at the center of four conducting rods with which we can vary the magnitude and direction of the electric field at the trap, thus controlling the orientation of the dipoles with respect to the sample axis. We have measured the strength of the interaction for a variety of relative orientations.
\end{abstract}

DOI: 10.1103/PhysRevLett.93.153001

PACS numbers: 32.80.Pj, 03.67.Lx, 32.80.Rm, 34.60.+z

A controlled interaction between quantum systems is a primary ingredient for quantum computation. While the coherent manipulation of a variety of coupled quantum systems is being pursued, the simplicity of atoms and ions and the precision with which they can be manipulated make them particularly attractive in these studies [1]. Recently, researchers were able to entangle four ions in a realization of a four-quantum-bit logic gate [2]. Although this is an impressive accomplishment, decoherence due to stray electric fields remains problematic in the ionic system. Several proposals have suggested ways in which neutral atoms may be entangled by exploiting the exaggerated properties of Rydberg states that are excited in a sample of trapped atoms to produce the strong, longrange interaction necessary to build a fast quantum gate [3-6].

Cold Rydberg atoms also present an avenue for studying fundamental physical processes. The time scale for a typical experiment in this system can vary from tens of nanoseconds to tens of microseconds and is set by the interaction strength among atoms and the lifetime of the Rydberg states. On these time scales atoms at typical temperatures and densities achievable in a magnetooptical trap (MOT) move only a small fraction of the average interatomic spacing. Such a nearly frozen gas of strongly interacting atoms behaves in many ways like an amorphous solid. In such a solid, the resonant energy exchange among atoms is not dominated by two body interactions, but rather by the collective interaction among many atoms in the sample [7-9]. Our goal in this work is to manipulate both the internal state of the atoms and the spatial structure of the sample to explore and control the interactions and energy transport in this system. As a first step toward this goal we have constructed a one-dimensional sample, which suppresses many body effects, and explored the angular dependence of the dipole-dipole interaction between atoms.
Our experiment begins by trapping about $10^{6}$ atoms in a MOT. Next we tightly focus a pulsed blue laser beam through the MOT to excite a line of Rydberg atoms. This excitation is done in the presence of a static electric field, which serves to tune the energies of the atoms so that they may resonantly interact as well as orient these interacting dipoles [10]. After a fixed interaction time the atoms are field ionized in order to measure the number of atoms that have resonantly interacted.

We trap ${ }^{85} \mathrm{Rb}$ atoms using diode lasers in a standard MOT [11-13]. The typical trap for this experiment had a diameter of $0.5 \mathrm{~mm}$ and contained about $10^{6}$ atoms at a temperature of approximately $300 \mu \mathrm{K}$. The MOT is centered in a configuration of four parallel field wires, arranged at the corners of a square of side $1.27 \mathrm{~cm}$.

To produce Rydberg atoms, the ${ }^{85} \mathrm{Rb}$ atoms are excited to the $32 d$ level from the $5 p_{3 / 2}$ state using a $480 \mathrm{~nm}$ dye laser (Coumarin-480) pumped with a frequency tripled Nd:YAG (Nd-doped yttrium aluminum garnet) laser running at $20 \mathrm{~Hz}$. The beam from the dye laser is spatially filtered and directed into the MOT along an axis perpendicular to the field wires. Using a doublet-meniscus lens combination, the beam is focused to a waist of approximately $10 \mu \mathrm{m}$. The excited volume of cold atoms is long (the diameter of the trap) and thin, with a diameter of approximately the same value as the interatomic spacing. This scheme produces a nearly one-dimensional line of Rydberg atoms thus defining the direction of the separation vector between atoms.

To control the orientation of the dipole moments relative to the separation vector, a static electric field is introduced. The voltage on each of the four rods is independently set to produce an electric field of the desired magnitude and direction at the trap. The electric field can be continuously scanned in magnitude over a $2.5 \mathrm{~V} / \mathrm{cm}$ range while keeping its direction fixed. 
The atoms are allowed to interact in the static electric field for $3 \mu \mathrm{s}$. This is long enough to allow for an appreciable energy transfer, but short compared to the Rydberg state lifetime of $37 \mu \mathrm{s}$. This $3 \mu \mathrm{s}$ interaction time is also short enough that a typical atom in our MOT moves at most one tenth of the average interatomic spacing. At various electric field strengths, there are Stark states equally spaced in energy above and below the excited $d$ state, where a resonant energy exchange can occur. There are two sets of states for which this happens,

$$
\begin{aligned}
& 32 d+32 d \rightarrow 34 p+(n=30) \quad \text { and } \\
& 32 d+32 d \rightarrow 33 p+(n=31),
\end{aligned}
$$

where $(n=30)$ and $(n=31)$ refer to manifold states. Figure 1 shows a Stark map for the region around $32 d$ along with a typical resonance of the type described by Eq. (1a). The energy exchange between two Rydberg atoms in the MOT is mediated by the dipole-dipole interaction,

$$
V=\frac{\boldsymbol{\mu}_{1} \cdot \boldsymbol{\mu}_{2}-3\left(\boldsymbol{\mu}_{1} \cdot \hat{\mathbf{R}}\right)\left(\boldsymbol{\mu}_{2} \cdot \hat{\mathbf{R}}\right)}{R^{3}} .
$$

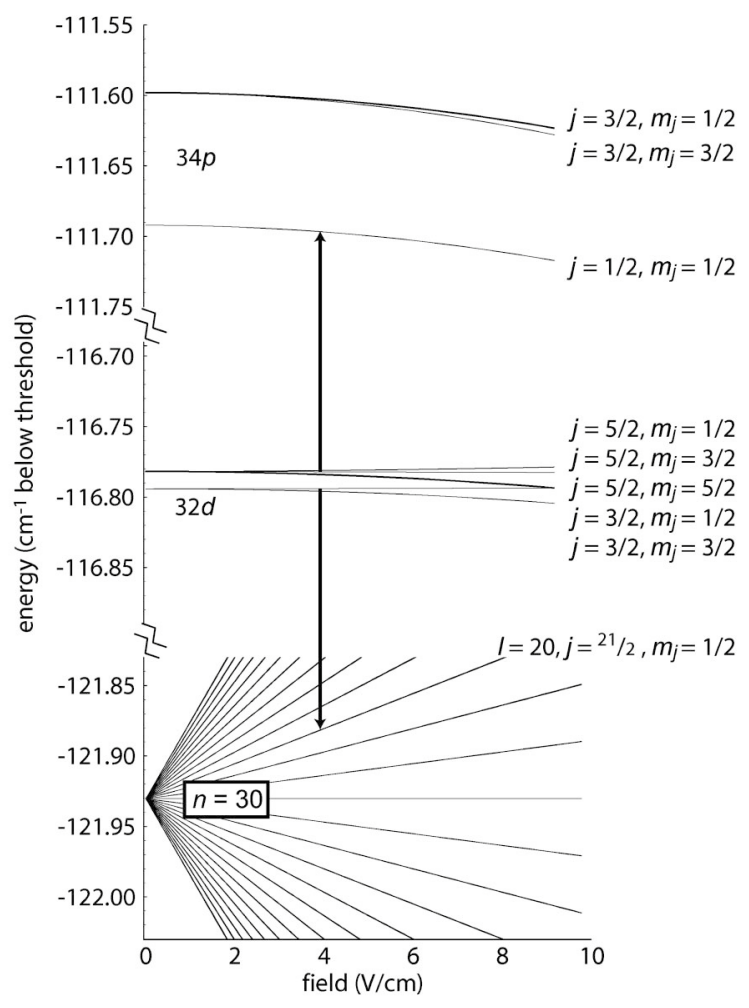

FIG. 1. Stark map of the $32 d$ states, $34 p$ states, and $n=30$ manifold states that contribute to the resonances of Eq. (1a). For clarity, only the $m_{j}=1 / 2$ states are shown for the manifold. The example resonance shown depicts two interacting atoms that have been excited to the $32 d_{3 / 2}, m_{j}=1 / 2$ and the $32 d_{5 / 2}, m_{j}=3 / 2$. One atom is further excited to the $34 p_{1 / 2}$ while the other falls to a manifold state. Both the $34 p$ and $32 d$ states are detected using selective field ionization.
Here $\boldsymbol{\mu}_{1}$ and $\boldsymbol{\mu}_{2}$ are the $32 d \rightarrow 34 p$ and $32 d \rightarrow(n=30)$ electric dipole matrix elements and $\mathbf{R}$ is the separation vector connecting the pair of atoms [14].

At the end of the interaction time a high voltage field ionization pulse is applied to the pair of wires furthest from the electron detector. The pulse amplitude is adjusted to ionize the initial $32 d$ state along with any atoms that have been excited to the $34 p$ state by interacting via Eq. (1a). The ionized electrons are detected with a chevron microchannel plate assembly. The field ionization pulse has a rise time of $1.5 \mu \mathrm{s}$ and provides sufficient energy resolution to time resolve the $32 d$ signal from the $34 p$ signal, but not to resolve the $j$ or $m_{j}$ sublevels of these states. The interactions of Eq. (1b) occur at a higher field and lie outside of our current scan range.

The time resolved electron signal is collected as the magnitude of the static electric field is scanned over many shots of the laser. The fraction of atoms that interacts is found by integrating the first peak in the time resolved signal, which is associated with the $34 p$ state, and normalizing by the total $(32 d+34 p)$ signal. In Fig. 2 we plot this fraction as the static field is scanned through many resonances. Because of the large number of possible states (all $j$ and $m_{j}$ values for the two initial $d$ states, the final $p$ state, and manifold states), there are approximately 5000 possible resonances in the region from 0 to $10 \mathrm{~V} / \mathrm{cm}$. The strongest of these resonances tend to cluster in a way that gives the appearance of a rather simple spectrum; however, each of the broad peaks seen in our data is actually a superposition of a number of resonances. The general agreement between the data and calculation shown in Fig. 2 demonstrates a basic understanding of the numerous interactions that occur in this range of fields. A full description of this calculation is given after we discuss angular dependence.

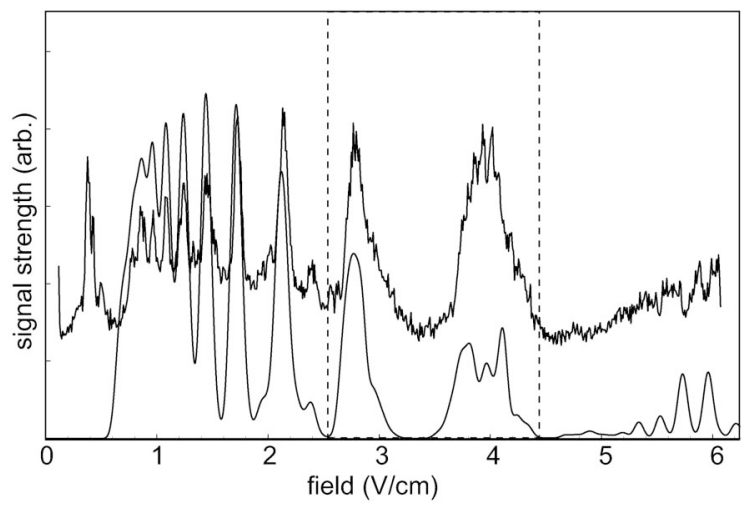

FIG. 2. Scan of the $32 d$ interaction superimposed on a calculation of the interaction. The calculation accounts for most of the major features, except for the peak near $0.5 \mathrm{~V} / \mathrm{cm}$, which may not be a result of the interactions of Eqs. (1a) and (1b). This scan was taken with the field oriented at $90^{\circ}$ with respect to the sample axis. The dashed line sets off our region of interest for the angular dependence study. 
In our exploration of the angular dependence of the dipole-dipole interaction we focus our attention on the two peaks that fall between 2.5 and $4.5 \mathrm{~V} / \mathrm{cm}$. We begin by carefully overlapping the focus of the dye laser with the MOT. This is done by translating the lens assembly along the laser's propagation direction to the position that minimizes the total electron signal. The minimum electron signal corresponds to the smallest excited atom volume, which occurs when the focus is at the center of the trap. The transverse position of the focus is also adjusted to be well centered within the electrode configuration to maximize the field homogeneity. Figure 3 shows the fraction of atoms that interacted for two different angles. The angle is measured between the applied electric field, which orients the dipoles, and the sample axis, which defines the separation vector. We see that the resonant interaction is much larger at $90^{\circ}$ than at $45^{\circ}$. For each angle, the total area of both peaks was integrated to measure the interaction strength at that angle. We also scan the field amplitude for each of seven other orientation angles and integrate the area under these peaks to produce the plot of interaction strength versus angle as shown in Fig. 4.

Modeling this data requires a careful consideration of all of the transitions that contribute to the interactions in these two peaks. We only model pairwise interactions between atoms. Many body effects were neglected because each atom in a one-dimensional sample has far fewer neighbors than in a three-dimensional amorphous solid. In particular, each atom in the line will have only two neighbors, one on each side, and the interaction will be dominated by the closer of the two. Our calculations begin with the generation of a Stark map for the region $n=26-43$ [15]. Using this Stark map, the locations of resonances are found (simply by searching for electric fields where the energy separations are equal). For each of these resonances, the matrix elements corresponding to

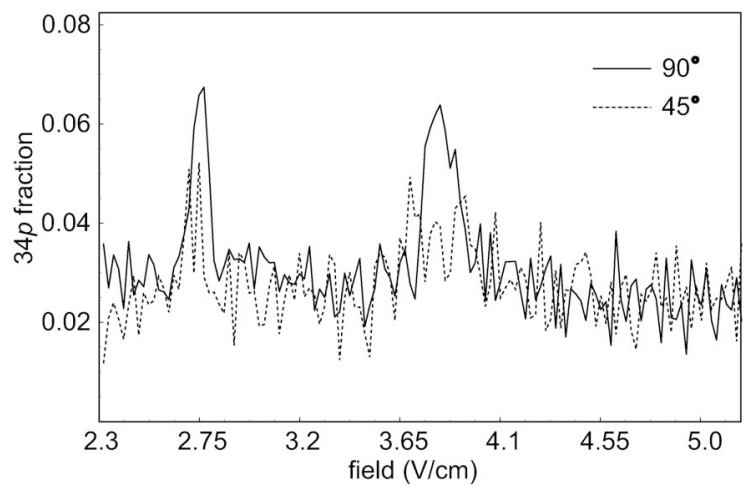

FIG. 3. Voltage scans at two angles for the two peaks that were studied, showing the fraction of atoms excited to the $34 p$ state. The solid line is a scan at a relative orientation angle of $90^{\circ}$, while the dotted line is for $45^{\circ}$. The total area beneath the peaks was used as a measure of the relative strength of the interaction. all of the possible $\Delta m_{j}$ transitions are calculated. It is in these matrix elements that the angular dependence appears. The matrix elements are calculated using

$$
V=\frac{e^{2}}{R^{3}}\left\langle\psi_{1}\left|\left\langle\psi_{2}\left|\mathbf{r}_{1} \cdot \mathbf{r}_{2}-3\left(\mathbf{r}_{1} \cdot \hat{\mathbf{R}}\right)\left(\mathbf{r}_{2} \cdot \hat{\mathbf{R}}\right)\right| \psi_{2}^{\prime}\right\rangle\right| \psi_{1}^{\prime}\right\rangle,
$$

where the subscripts refer to different atoms and the primed and unprimed states refer to the final and initial states, respectively, and $\boldsymbol{\mu}_{1}$ and $\boldsymbol{\mu}_{2}$ have been factored according to $\boldsymbol{\mu}=e \mathbf{r}$. Writing out the scalar products we obtain

$$
\begin{aligned}
V= & \frac{e^{2}}{R^{3}}\left\langle\psi_{1}\right|\left\langle\psi_{2}\right| x_{1} x_{2}+y_{1} y_{2}+z_{1} z_{2}-3\left(x_{1} \hat{\mathbf{R}}_{\mathbf{x}}+y_{1} \hat{\mathbf{R}}_{\mathbf{y}}\right. \\
& \left.+z_{1} \hat{\mathbf{R}}_{\mathbf{z}}\right)\left(x_{2} \hat{\mathbf{R}}_{\mathbf{x}}+y_{2} \hat{\mathbf{R}}_{\mathbf{y}}+z_{2} \hat{\mathbf{R}}_{\mathbf{z}}\right)\left|\psi_{2}^{\prime}\right\rangle\left|\psi_{1}^{\prime}\right\rangle,
\end{aligned}
$$

where

$$
\begin{gathered}
\hat{\mathbf{R}}_{\mathbf{x}}=\sin \theta \cos \phi \hat{\mathbf{x}}, \quad \hat{\mathbf{R}}_{\mathbf{y}}=\sin \theta \sin \phi \hat{\mathbf{y}}, \\
\hat{\mathbf{R}}_{\mathbf{z}}=\cos \theta \hat{\mathbf{z}} .
\end{gathered}
$$

The radial component of the integral is calculated using the Numerov method [16] while the terms in Eq. (5) account for the angular dependence of the matrix elements $[17,18]$. Averaging over $\phi$, there are three types of angular dependence present:

$$
\begin{gathered}
f_{1}=\left(1-3 \cos ^{2} \theta\right)^{2}, \quad f_{2}=9 \sin ^{2} \theta \cos ^{2} \theta, \\
f_{3}=9 \sin ^{4} \theta .
\end{gathered}
$$

In Eq. (6), $f_{1}$ is the expected angular dependence in the classical case that one would obtain using Eq. (2) for two aligned dipoles, which corresponds to a resonant energy exchange between states where $\Delta m_{j}=0$ for both atoms or where $\Delta m_{j}= \pm 1$ for one atom and $\Delta m_{j}=\mp 1$ for the other. The other types $\left(f_{2}\right.$ and $\left.f_{3}\right)$ arise from the different combinations of Eq. (5) present in the integrals and correspond to $\Delta m_{j}=0$ for one atom and $\Delta m_{j}= \pm 1$ for the other for $f_{2}$ or to $\Delta m_{j}= \pm 1$ for both atoms for an angular dependence given by $f_{3}$.

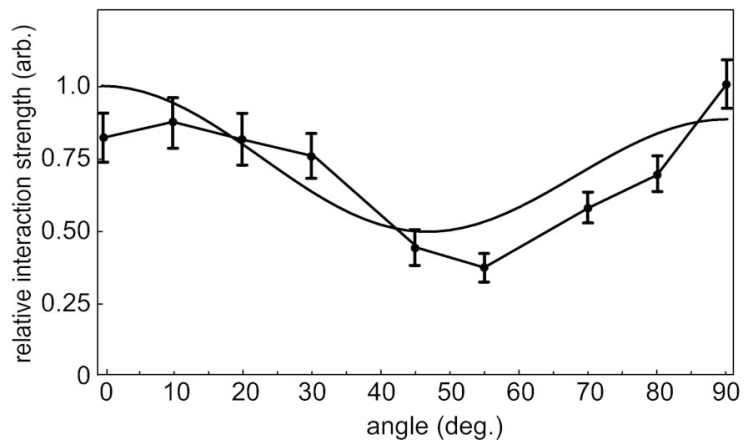

FIG. 4. Interaction strength versus angle in arbitrary units. Data were taken at nine different angles. The smooth curve is a calculated angular dependence, with no fitting parameters. The error bars represent statistical uncertainties in our measurements. 
Once all of the matrix elements are calculated, they are squared to give an intensity for each resonance. Returning to Fig. 2 we show the results of these calculations when each resonance is assigned a width of $0.04 \mathrm{~V} / \mathrm{cm}$ based on comparison to the experimental data. The initial population of excited states is calculated by finding the oscillator strengths from the $5 p_{3 / 2}\left(m_{j}=\right.$ $1 / 2, m_{j}=3 / 2$ ) states to the various $32 d$ states. This is a reasonable assumption given that our dye laser bandwidth is too broad to resolve the fine structure splitting of the $32 d$ state. Figure 2 shows good agreement between our experimental and theoretical results for the fields at which the resonant energy exchanges occur; however, there is some discrepancy in the relative strengths of these interactions. Given the simplicity of our calculation these discrepancies are not surprising. In fact, this model is a measure of the interaction strength between a pair of atoms and does not take into account any residual many body effects or the details of the time dependence of the interaction.

To calculate the theoretical angular dependence curve shown in Fig. 4, the area under each of the two peaks we studied is calculated for a range of angles. While the agreement between the theoretical and experimental curves is not exact, there is strong qualitative agreement. The angular dependence shows a broad minimum near $55^{\circ}$ in the measured signal strength and near $45^{\circ}$ in the calculated interaction strength. Likely sources for this discrepancy are the simplicity of our model along with the complexity of the system in terms of the number of states that can resonantly exchange energy in this region. By controlling the initial excited states present or by finding a spectral region where the resonances are better isolated, one should be able to isolate the three angular dependencies of Eq. (6). More precise control of this type may ultimately be useful in engineering controlled quantum interactions useful for quantum computing.

The angular dependence observed in our onedimensional sample of atoms also has important implications for the energy transfer in the three-dimensional amorphous systems previously studied, which point to the importance of many body interactions among atoms. It has been suggested that the simultaneous interaction among many atoms in the three-dimensional amorphous sample may lead to the formation of much broader energy bands than would be seen if two body collisions were dominant [7]. Recent dephasing measurements support this notion [19]. In another model of this amorphous sample, the energy exchange is initiated between close pairs of atoms and then diffuses away in analogy to an autocatalytic process in a chemical reaction [8]. In either case, all of the dipole moments in the system are initially aligned by the applied static field. The angular dependence of the interaction will then limit the dimensionality or pathway of the many body interactions. Further studies of how the dimensionality and spatial structure of the cold Rydberg atom sample can affect the energy exchange among atoms are underway.

It is a pleasure to acknowledge helpful discussions with T. F. Gallagher during the course of this work. This work was supported by the National Science Foundation under Grant No. 0134676. Funding for the 24-node Beowulf cluster on which the calculations were run was provided by Bryn Mawr College.

*Also at: Physics Department, Harvard University, Cambridge, MA 02138, USA.

†Present address: Department of Physics and Astronomy, Rowan University, Glassboro, NJ 08028, USA.

[1] S. L. Braunstein and H. K. Lo, Scalable Quantum Computers: Paving the Way to Realization (Wiley, New York, 2000).

[2] C. A. Sackett et al., Nature (London) 404, 256 (2000).

[3] D. Jaksch, J. I. Cirac, P. Zoller, S. L. Rolston, R. Côté, and M. D. Lukin, Phys. Rev. Lett. 85, 2208 (2000).

[4] M. D. Lukin, M. Fleischhauer, R. Cote, L. M. Duan, D. Jaksch, J. I. Cirac, and P. Zoller, Phys. Rev. Lett. 87, 037901 (2001).

[5] I. E. Protsenko, G. Reymond, N. Schlosser, and P. Grangier, Phys. Rev. A 65, 052301 (2002).

[6] M. S. Safronova, C. J. Williams, and C.W. Clark, Phys. Rev. A 67, 040303(R) (2003).

[7] W. R. Anderson, J. R. Veale, and T. F. Gallagher, Phys. Rev. Lett. 80, 249 (1998).

[8] I. Mourachko, D. Comparat, F. de Tomasi, A. Fioretti, P. Nosbaum, V. M. Akulin, and P. Pillet, Phys. Rev. Lett. 80, 253 (1998).

[9] J. S. Frasier, V. Celli, and T. Blum, Phys. Rev. A 59, 4358 (1999).

[10] D. S. Thomson, R. C. Stoneman, and T. F. Gallagher, Phys. Rev. A 39, 2914 (1989).

[11] E. L. Raab, M. Prentiss, A. Cable, S. Chu, and D. E. Pritchard, Phys. Rev. Lett. 59, 2631 (1987).

[12] D. W. Sesko, T. Walker, and C. Wieman, J. Opt. Soc. Am. B 8, 946 (1990).

[13] C. D. Wallace, T. P. Dinneen, K. Y. N. Tan, A. Kumarakrishnan, P. L. Gould, and J. Javanainen, J. Opt. Soc. Am. B 11, 703 (1994).

[14] T. F. Gallagher, Rydberg Atoms, Cambridge Monographs on Atomic, Molecular, and Chemical Physics (Cambridge University, Cambridge, England, 1994).

[15] M. L. Zimmerman, M. G. Littman, M. M. Kash, and D. Kleppner, Phys. Rev. A 20, 2251 (1979).

[16] Computational Physics, edited by S. E. Koonin and D. C. Meredith (Addison-Wesley, Reading, MA, 1990).

[17] J. S. Frasier, Ph.D. thesis, University of Virginia, 2001.

[18] H. A. Bethe and E. E. Salpeter, Quantum Mechanics of One- and Two-Electron Atoms (Plenum, New York, 1977).

[19] W. R. Anderson, M. P. Robinson, J. D. D. Martin, and T. F. Gallagher, Phys. Rev. A 65, 063404 (2002). 